MODELING, IDENTIFICATION AND CONTROL, 1988, VOL. 9, NO. 1, 47-56

doi:10.4173/mic.1988.13

\title{
Estimating the condition of the heat resistant lining in an electrical reduction furnace
}

\author{
JAN GUNNAR WAALMANN $\dagger$
}

Keywords: Modeling, estimation, condition monitoring, heat resistant materials, process equipment, ferrosilicon.

This paper presents a system for estimating the condition of the heat resistant lining in an electrical reduction furnace for ferrosilicon. The system uses temperature measured with thermocouples placed on the outside of the furnace-pot. These measurements are used together with a mathematical model of the temperature distribution in the lining in a recursive least squares algorithm to estimate the position of 'the transformation front'. The system is part of a monitoring system which is being developed in the AIP-project: "Condition monitoring of strongly exposed process equipment in the ferroalloy industry'. The estimator runs on-line, and results are presented in colour-graphics on a display unit. The goal is to locate the transformation front with an accuracy of $\pm 5 \mathrm{~cm}$.

\section{Introduction}

'Condition monitoring of strongly exposed process equipment in the ferroalloy industry' is a project which is part of a research programme entitled 'Automation in the process industry' (AIP) which is sponsored by the Royal Norwegian Council for Scientific and Industrial Research, and consists of eight projects in the field of monitoring and control. The condition monitoring project we are responsible for is a joint venture between Elkem a/s and Institute for Energy Technology (IFE).

During the recent years, the ferroalloy industry has invested a lot of time and money in automation and process improvements. This has resulted in optimizing operations, provided the process equipment functions properly. At the same time there is a trend towards planned, preventative maintenance of the equipment. The reason for this new interest in maintenance is that preventative maintenance enables the operating time of the furnace to be increased, by avoiding unforeseen halts.

The goal of the project is to develop a condition monitoring system for an open, electrical reduction furnace. Our test object is furnace no. 3 at Elkem a/s, Salten Verk. We have planned to monitor these five types of equipment (Fig. 1): Furnacepot, electrical supply system, smoke hood, electrodes and raw material transportation. This paper presents part of the monitoring system for the furnace-pot.

\section{The problem}

A reduction furnace for ferrosilicon includes a furnace-pot which consists of a steel jacket lined with heat resistent materials. Typical dimensions are a diameter of about $10 \mathrm{~m}$, and a weight (lining materials only) of about 500 tons.

Received September 111987.

† Institute for Energy Technology, P.O. Box 40, N-2007 Kjeller, Norway. 


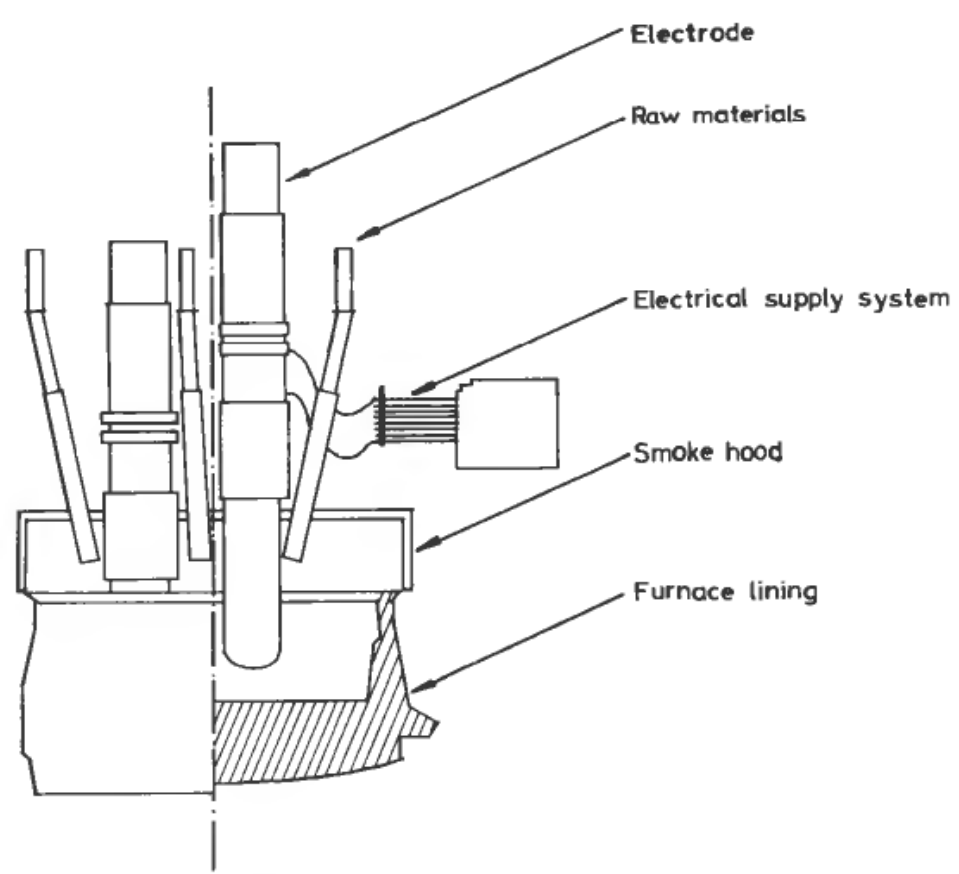

Figure 1. An open reduction furnace.

The materials in the lining are exposed to hard wear when the process is operational. First, the temperature in the molten metal is approximately $1800^{\circ} \mathrm{C}$. Then, during production, $\mathrm{SiO}$ is released and some of it penetrates the heat resistant materials. At the same time the molten metal seeps down into every crack in the lining. The result is that the heat resistant materials are transformed and partly replaced by molten metal.

When examining old linings, a relatively well defined boundary is frequently found between the transformed and the untouched parts. This boundary, 'the transformation front', usually has a parabolic shape. The lowest temperature, at which one can find molten ferrosilicon, is $1207^{\circ} \mathrm{C}$ (eutectic temperature), and the transformation front is often called 'the 1200 degrees isotherm'. This name is due to the assumption that one can find molten metal all the way down to the transformation front. Although it is an oversimplification, one can say that there is a well defined boundary, above which the material is transformed while the material below remains intact.

The problem of uncontrolled bottom-drainage has recently become more acute because optimized operations, with fewer and shorter stops, exposes the lining to harder wear than before. Previously there were frequent stops which lasted longer than they do today. During such periods, the metal in the lining solidified and formed a barrier of solid metal. This made the lining stronger, because much more energy was required to melt the solid metal. Today this process runs continuously, and the transformation takes place in a shorter period of time. Better knowledge about how to operate the furnace, also results in a more accurate positioning of the electrodes. Quite often this means that the electrodes are positioned lower than before, and consequently the thermal wear upon the lining is increased.

The use of preventative maintenance to avoid bottom-drainages will improve both safety and economy. However, it is problematic to decide when the lining has 


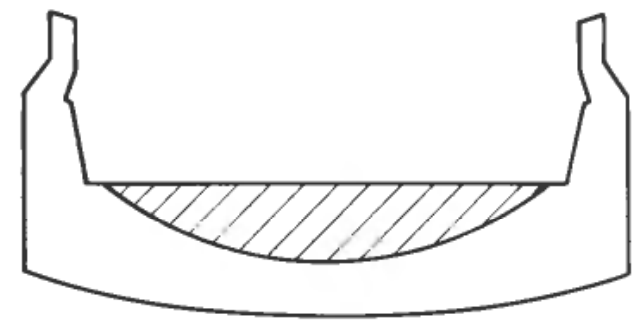

Figure 2. Lining and transformation front.

to be rebuilt. One way of determining this matter, is to decide that the lining should be rebuilt when the transformation front reaches some prespecified distance from the bottom of the lining. A system for condition monitoring should be able to act as a maintenance tool and estimate the position of the transformation front. This leaves the problem of how to give a good estimate of the shape and position of the transformation front, based solely on temperature-measurements made on the outside of the furnace-pot.

The furnace-pot is equipped with 31 thermocouples to measure the surface temperature and 4 thermocouples to measure the air temperature. The estimator uses 13 of the surface temperatures and all of the air temperatures. The measurements are transmitted to the dedicated computer by means of an optical communication channel. The optical fibre has proved itself very useful in the environment close to an electrical furnace.

In addition to the estimator, the monitoring system includes ordinary monitoring which uses all of the measurements. This monitoring system includes graphical presentation, an alarm check and a trend check.

\section{Modeling}

A relatively simple three-dimensional mathematical model has been constructed. The model describes the temperature distribution in the bottom of the heat resistant furnace-lining. The distribution depends on the shape and position of the transformation front.

We have chosen a model description using what is often called 'the heat balance method' (Welty, 1974). In this description the lining is divided into a number of computational elements, 'boxes'. Each element is described by mean values (temperature and thermal conductivity). Heat transfer is calculated between node points situated in the centre of each box. This description is mainly chosen because the model is computed on-line, and we want to reduce the computational time as much as possible. It is sufficient to use a static description because the transformation process is very slow.

Examinations of old linings have led us to describe the transformation front as a paraboloid (Fig. 2). Since the furnace has a cylindrical shape, these two factors have made us opt for a model which is symmetrical around the central axis of the lining (rotational symmetry). We have used cylindrical coordinates with the centre of the system of coordinates situated on the central axis of the lining. The positive $z$ direction is downwards. In our description, the lining is divided into 21 'boxes'. This number follows the natural arrangement in layers (7 layers including the 


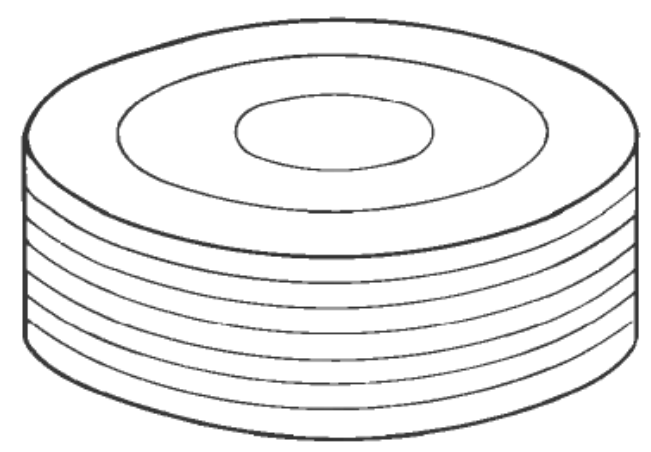

Figure 3. Lining divided into 21 'boxes'.

jacket), and divides each layer into three boxes (Fig. 3). This division ensures that there is a simple relationship between the measured and calculated values.

It is now possible to establish a static heat balance equation for every box. The boxes being represented by their mean values and the heat transfer calculated between node points in the centre of each box. For an arbitrary box, the equation will be:

$$
\begin{gathered}
q_{\text {from above }}+q_{\text {from inside }}-q_{\text {to below }}-q_{\text {to outside }}=0 \\
q-\text { Heat transfer rate }
\end{gathered}
$$

This produces a system of 21 equations. To speed up the computations these equations have been linearized, and become in matrix form:

$$
A \mathbf{x}=\mathbf{b}
$$

\section{$A$ Coefficient matrix}

$x$ Vector containing the 21 temperatures

b Vector

The heat transfer between two boxes follows Fourier's heat rate equation:

$$
q=-\frac{\lambda A \Delta T}{l}
$$

$\lambda$ Thermal conductivity

$A$ Area of the boundary normal to the direction of the flow

$\Delta T$ Difference between mean temperatures

$l$ Distance between node points

Providing the thermal conductivity is the same, the heat transfer between the two outermost boxes in a layer (heat transfer in a cylinder from radius $r_{0}$ to $r_{1}$ ) may be written (Wong, 1977):

$$
q=\lambda 2 \pi h \frac{\left(T_{0}-T_{1}\right)}{\ln \left(r_{1} / r_{0}\right)}
$$

$h$ Height of the boundary between the boxes.

The model has three boundaries, each of these having its own boundary-conditions. The uppermost layer in the lining receives heat transferred from the molten metal. We assume that the thermal conductivity in the metal is high enough to assure that 
the surface-temperature of the uppermost layer equals the metal temperature. At the boundary underneath the furnace-pot, the heat is transferred by a combination of convection and radiation. The convective heat transfer is expressed by Newton's rate equation:

$$
q=h_{c} A\left(T_{\text {surface }}-T_{\text {air }}\right)
$$

$h_{c}$ Convective heat transfer coefficient

\section{$A$ Surface area}

The radiative heat transfer is expressed by the Stefan-Bolzmann radiation law. Since this is a non-linear expression, it has been linearized around the last calculated surface temperature. This means that the linearization point changes from iteration to iteration. When linearized around a fixed surface temperature, the expression may be written (for a grey body):

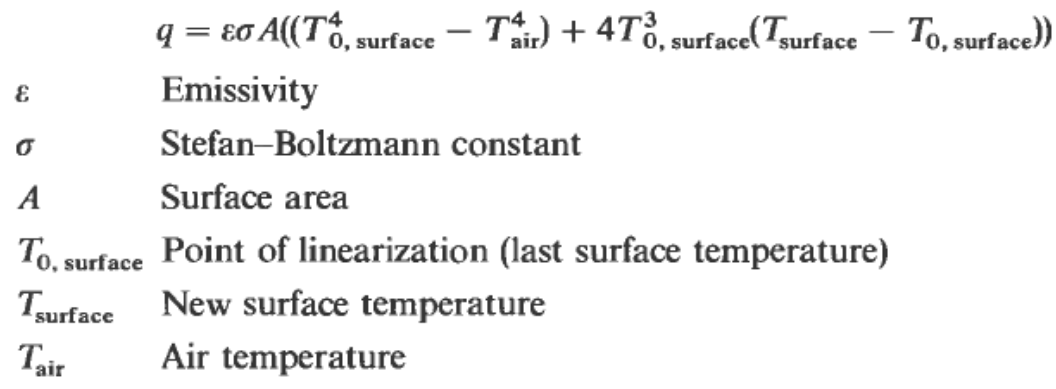

Heat is also transferred through the wall of the lining. We use a simplified description of this wall, in which the wall has rotational symmetry. We also assume that there is no vertical heat transfer in the wall. The heat is transferred from the wall by a combination of convection and radiation. We use the same expressions as we did on the boundary underneath the pot. With a finned surface, the total heat transfer may be expressed as:

$$
q=\left(2 \pi r_{y} h+\eta_{f} A_{f}\right)\left(h_{c}\left(T_{y}-T_{\infty}\right)\right)+2 \pi r_{y} h \varepsilon \sigma\left(T_{0, y}^{4}-T_{\infty}^{4}+4 T_{0, y}^{3}\left(T_{y}-T_{0, y}\right)\right)
$$

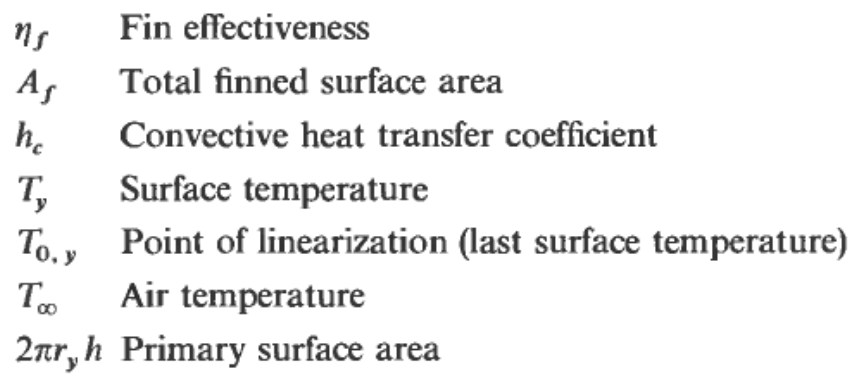

In the model, the connection between the transformation front and the temperaturedistribution is described using thermal conductivities and volume computations. In the chosen system of coordinates, the transformation front may be written as a paraboloid:

$$
z=-a r^{2}+b
$$

Given values for $a$ and $b$, the front will cut the boxes in a specific way (Fig. 4). It is a comparatively simple task to calculate the transformed and untouched volume of every box. The transformed part will have a new, high conductivity, while the 


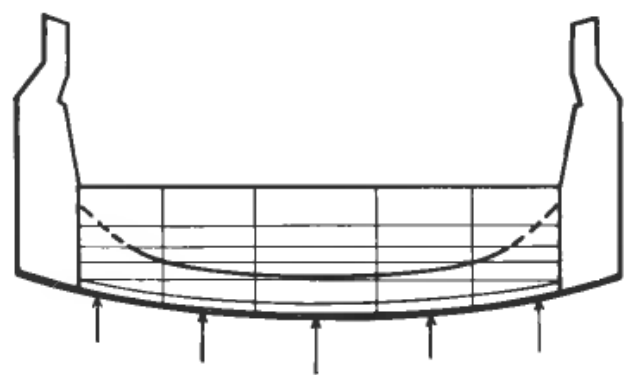

Figure 4. The transformation front cutting through the boxes.

untouched part retains the original conductivity. The original thermal conductivity of heat resistant stone may be $1.25 \mathrm{~W} / \mathrm{mK}$, while the conductivity of the transformed material may be $6.0-8.0 \mathrm{~W} / \mathrm{mK}$.

As an example we can compute the volume restricted by $z=-a r^{2}+b, z=z_{0}$, $r=r_{i}$ and $r=r_{y}$ :

$$
\begin{aligned}
V & =\int_{r_{i}}^{r_{y}} 2 \pi r\left(-a r^{2}+b-z_{0}\right) d r \\
& =\pi\left(\left(b-z_{0}\right)\left(r_{y}^{2}-r_{i}^{2}\right)-\frac{a}{2}\left(r_{y}^{4}-r_{i}^{4}\right)\right)
\end{aligned}
$$

As the transformation front may cut through a box in a number of ways, the computation of the volumes must take this into consideration. We have designed a dedicated module to solve this problem and allow us to choose the correct volume expression.

Given $a$ and $b$, the volume expressions are chosen, the volumes are then calculated, and new mean conductivities are computed as functions of the volumes. In this way mean conductivities are functions of $a$ and $b$. The calculated conductivities form part of the model equations, and a new temperature-distribution can be computed. The temperatures of the surface underneath the furnace-pot are among the computed temperatures. These surface temperatures are the same as the ones we measure, and the deviations from the measurements form a basis for estimating $a$ and $b$.

The model equations, which form a system of 21 linear equations, are then solved using the Gauss elimination with pivoting.

\section{Estimation}

We have designed an algorithm for estimating the shape and position of the transformation front (Fig. 5). The algorithm estimates the parameters in Eqn (1). Based on measured and calculated values, we can construct a criterion function:

$$
F=p_{1}(\hat{a}-\bar{a})^{2}+p_{2}(\hat{b}-\bar{b})^{2}+q_{1}\left(T_{1}-y_{1}\right)^{2}+q_{2}\left(T_{2}-y_{2}\right)^{2}+q_{3}\left(T_{3}-y_{3}\right)^{2}
$$



$\hat{a}$ New estimated value
$\bar{a}$ Old estimated value
$y_{i}$ Measured values
$T_{i}$ Calculated values
$p_{i}$ Weighting factor
$q_{i}$ Weighting factor

To find the optimal choice of the parameters, we minimize the criterion with respect to $a$ and $b$. Minimizing the criterion means minimizing a weighted sum of (squared) residuals between measured and calculated values, as well as the deviations between the old and the new estimates

The measurements are filtered through a lowpass filter before entering the estimation algorithm. This means that they vary very little, and consequently the new estimates more or less equal the old ones. Taking this into consideration, we can rewrite the criterion function:

$$
F=q_{1}\left(T_{1}-y_{1}\right)^{2}+q_{2}\left(T_{2}-y_{2}\right)^{2}+q_{3}\left(T_{3}-y_{3}\right)^{2}
$$

This can be generally expressed:

$$
\begin{aligned}
F & =f_{1}^{2}+f_{2}^{2}+f_{3}^{2}=\mathbf{f}^{\mathrm{T}} \mathbf{f} \\
\mathbf{f}^{\mathrm{T}} & =\left[f_{1}, f_{2}, f_{3}\right]
\end{aligned}
$$

This criterion forms a basis for the least squares estimation algorithm. Minimizing the criterion function means searching for the zeroes of the derivatives of the function with respect to $a$ and $b$. The criterion is a function of the two parameters, $a$ and $b$, but the functional relationship is implicit not explicit. This means that we have to use numerical approximations for the derivatives. We have chosen to use a central difference representation of the derivatives:

$$
\begin{aligned}
\frac{\delta F}{\delta a} & \approx \frac{F(a+\Delta, b)-F(a-\Delta, b)}{2 \Delta} \\
\frac{\delta F}{\delta b} & \approx \frac{F(a, b+\Delta)-F(a, b-\Delta)}{2 \Delta}
\end{aligned}
$$

The fact that the functional relationship is implicit, means that the model-equations must be solved every time we want to calculate the value of the criterion for a given value of $a$ and $b$.

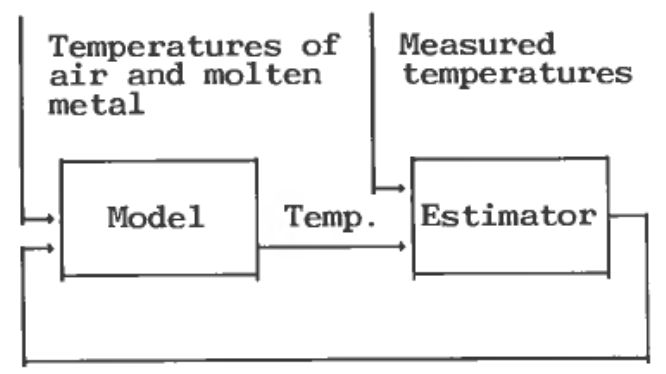

Parameters

Figure 5. Mathematical model and estimator. 


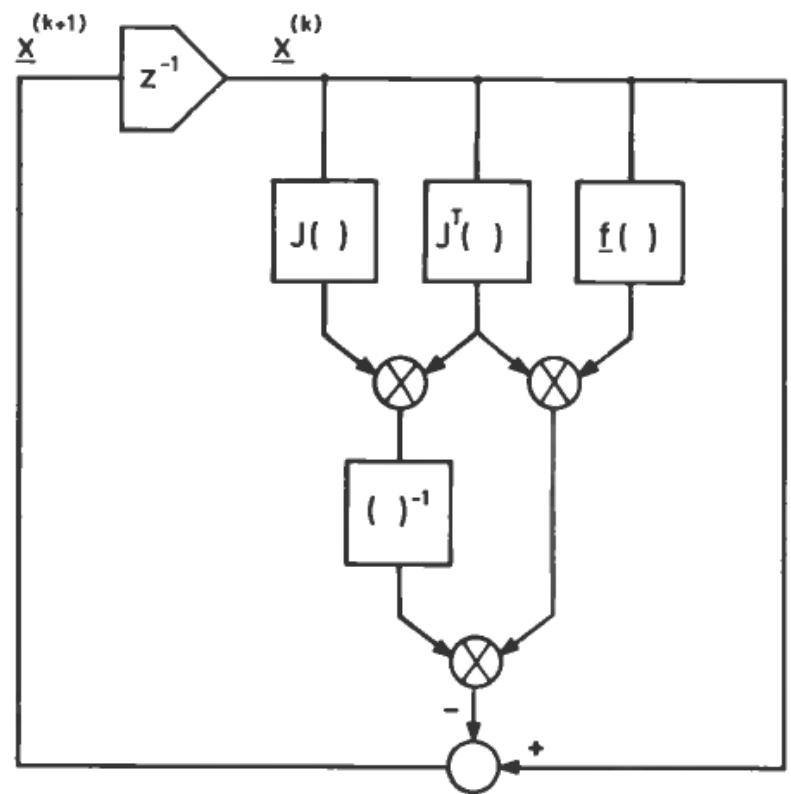

Figure 6. The Gauss-Newton algorithm.

In order to find the zeros of the derivatives, a recursive algorithm must be used. If we choose an ordinary Newton-Raphson method, we will have to establish approximations for the second order derivatives. When only single precision calculations are made, these approximations may introduce large numerical errors (especially for small values of $\Delta$ ). To avoid these problems, we have chosen to use the Gauss-Newton method. This algorithm uses derivatives of only the first order. The algorithm is very well suited when the criterion function is a sum of squares. In this algorithm, the Hessian, which consists of derivatives of $F$ of the second order, is expressed using the Jacobian which only contains first order derivatives of $f$. The approximation used is:

$$
G(\mathbf{x}) \approx 2 J(\mathbf{x})^{\mathrm{T}} J(\mathbf{x})
$$

(Dixon, 1976)

$G(\mathbf{x})$ Hessian

$J(\mathbf{x})$ Jacobian

If, for the sake of simplicity, we form a vector of the two parameters,

$$
\mathbf{x}=\left[\begin{array}{l}
x_{1} \\
x_{2}
\end{array}\right]=\left[\begin{array}{l}
a \\
b
\end{array}\right]
$$

the Gauss-Newton algorithm can be expressed in this form:

$$
\mathbf{x}^{(k+1)}=\mathbf{x}^{(k)}-\left(J\left(\mathbf{x}^{(k)}\right)^{\mathrm{T}} J\left(\mathbf{x}^{(k)}\right)\right)^{-1} J\left(\mathbf{x}^{(k)}\right)^{\mathrm{T}} \mathbf{f}\left(\mathbf{x}^{(k)}\right)
$$

The elements in the Jacobian are approximated using the central differences (Eqn 2). The iterative algorithm can be diagrammatically expressed as in Fig. 6. Each calculation of the Jacobian means that the model equations must be solved four times. The reason for this is that the derivatives must be calculated, which involves temperatures for four different values of $a$ and $b:(a+\Delta, b),(a-\Delta, b),(a, b+\Delta)$ and $(a, b-\Delta)$. 
The restricted computational accuracy may cause the iterative algorithm to calculate steps that are too long, and pass the minimum point. To avoid this we can modify the Gauss-Newton algorithm, using a Fibonacci division method (Schwefel, 1981). The Fibonacci division method is a one-dimensional search strategy. This interval division strategy requires an initial interval, containing the minimum point, and a number specifying the number of divisions to be performed. The initial interval is divided into smaller sub-intervals a predefined number of times, with the last interval always containing the minimum. This means that the minimum is located with an accuracy given by the size of the initial interval and the number of divisions. The name of the method comes from a series called the Fibonacci series.

Every time the ordinary Gauss-Newton algorithm has calculated a new point, the Fibonacci algorithm searches for a minimum in the interval formed by the old and the new Gauss-Newton point. This minimum point is taken to be the new Gauss-Newton point.

The search is terminated when one or more of the following three convergence criteria is satisfied:

1. $F<F$-value

The value of the criterion function is less than a given value.

2. $\left|\frac{\delta F}{\delta a}\right|<D 1$-value and $\left|\frac{\delta F}{\delta b}\right|<D 1$-value

The absolute value of the derivatives is less than a given value.

3. $|\hat{a}-\bar{a}|<a$-value and $|\hat{b}-\bar{b}|<b$-value and

$\left|\frac{\delta F}{\delta a}\right|<D 2$-value and $\left|\frac{\delta F}{\delta b}\right|<D 2$-value

The absolute deviation between the old and the new iterative values is less than the $a$-value and the $b$-value, and the absolute value of the derivatives is less than a given value ( $D 2$-value $>D 1$-value).

Convergence means that the search is terminated, and the new estimates are written to the system database.

\section{Results}

The estimator is part of a condition monitoring system for an open, electrical reduction furnace. The condition of the heat resistant furnace-lining is described by two parameters. These parameters describe the shape and position of the transformation front, which are estimated on-line. For testing purposes the estimator is run every second hour, although this is more frequent than absolutely necessary.

The system described above, with a linear model and a modified Gauss-Newton algorithm, is the second version of the system. The first version contained a nonlinear model and an ordinary Newton-Raphson algorithm. That version involved too much computational time, and the approximations used for the second order derivatives introduced some numerical problems. The new version has only been running continuously for a short period of time because of hardware problems. This means that it is too early to make any firm statements about the performance of the estimator, though it looks promising. 
The result of running through the system is that the two parameters, $a$ and $b$, and the temperature-distribution is calculated. These results are presented to the operator on a graphical colour display unit. The picture on this unit shows a cross section of the heat resistant furnace-lining with temperatures and the transformation front included.

One of the main problems concerning the accuracy of the estimator, is the initial condition. Furnace no. 3 at Salten Verk is approximately 10 years old, and the initial condition when starting the estimator is not entirely known. This means that our initial values are based on measurements and computations. The accuracy of our estimates are not restricted by the mathematical performance of the estimator, rather by the degree of uncertainty connected to the initial situation and some of the physical parameters in the model. A final judgement can only be made when the lining is taken apart.

\section{Conclusions}

The paper describes the development of a mathematical model of the temperature-distribution in the heat resistant lining of an electrical reduction furnace, and a least squares algorithm for the estimation of the transformation front in the lining. The estimator has been run on-line on a furnace at Elkem a/s, Salten Verk. The estimator has been developed as part of a system for condition monitoring, it can only describe the average situation, and cannot detect any local malfunctioning in the lining.

The total monitoring system can be utilized by various groups of users: operators, process engineers and maintenance personnel. The estimator, on the other hand, is mainly intended for use by the last two groups, and may be of limited use in the everyday operation of the furnace.

The first version of the estimator was installed in December 1986. The performance of this version was unsatisfactory and a new, revised version was installed in March 1987. The problems we experienced with the first version (numerical problems and long computational time), seem to be eliminated. However, experience with the new version is somewhat limited because hardware problems have prevented us running the estimator for long periods of time.

Since the temperature measurements are made on the outside of the furnace-pot, these measurements will be affected by thermal disturbance. Nevertheless, these fluctuations do not seem to affect the accuracy of the estimator.

\section{ACKNOWLEDGMENTS}

The author would like to thank Jon Brinch and Terje Leidal at Elkem and Peter Borg at Institute for Energy Technology for their contributions in developing the estimator and Elkem a/s for permission to publish this paper.

\section{REFERENCES}

Dixon, L. C. W. (1976). Optimization in Action (Academic Press), p. 5.

SCHWEFE, H. P. (1981). Numerical Optimization of Computer Models (John Wiley \& Sons), pp. 27, 29.

Welty, J. R. (1974). Engineering Heat Transfer (John Wiley \& Sons), pp. 85, 88.

WoNG, H. Y. (1977). Heat Transfer for Engineers (Longman), p. 19. 